\author{
XIII SIMPÓSIO NACIONAL DE SISTEMAS PREDIAIS \\ DESEMPENHO E INOVAÇÃO \\ DE SISTEMAS PREDIAIS HIDRÁULICOS \\ SÃO PAULO - 04 DE OUTURO DE 2019
}

\title{
APLICABILIDADE DAS NORMAS INTERNACIONAIS PARA A SELEÇÃO DE PARÂMETROS DE DIMENSIONAMENTO DO SISTEMA SIFÔNICO DE DRENAGEM DE ÁGUAS PLUVIAS NO BRASIL - PESQUISA DOCUMENTAL
}

\section{THE ROLE OF INTERNATIONAL STANDARDS FOR DIMENSIONING OF SIPHONIC ROOF DRAINAGE SYSTEMS - A LITERATURE REVIEW}

\author{
RICHERS, Sabine ${ }^{1}$; SOWMY, Daniel ${ }^{2}$ \\ ${ }^{1}$ Instituto de Pesquisas Tecnologicas (IPT),adm@ @isors.com.br \\ ${ }^{2}$ Universidade de São Paulo (Poli - USP), dss@usp
}

\begin{abstract}
RESUMO
O objetivo deste artigo é apresentar informações sobre as normas internacionais que poderão colaborar na elaboração de uma norma brasileira sobre Sistema Sifônico de Drenagem de Águas Pluviais (SSDAP). A pesquisa documental permitiu identificar as normas internacionais existentes possibilitando seu estudo comparativo. Os autores concluem que o projeto de um Sistema Sifônico é composto de quatro grupos de informações: (i) informações da edificação; (ii) cálculos preliminares; (iii) dimensionamento do projeto e (iv) informações detalhadas para a instalação. Os seis principais parâmetros de dimensionamento apresentados neste artigo, sobre normas e diretrizes internacionais, poderão colaborar na elaboração de uma norma brasileira sobre SSDAP. A importância deste estudo se deve ao fato dele ser o primeiro exclusivamente sobre o SSDAP publicado no Brasil.
\end{abstract}

Palavras-chave: Parâmetros, Dimensionamento, Sistema Sifônico.

\begin{abstract}
This paper is to present information about the international standards that could supply parameters to a future Brazilian standard of Siphonic Roof Drainage System. The documentary review allowed the identification of international standards enabling a comparative study. The authors concluded that the design of siphonic system should be show four groups of information: (i) information about the building; (ii )preliminary calculations; (iii) project design and (iv) detailed installation information.
\end{abstract}

Keywords: Standards, Design, Siphonic Systems. 


\section{INTRODUÇÃO}

O Brasil registrou nas últimas décadas um acentuado crescimento de empreendimentos industriais e comerciais. O Sistema Sifônico de Drenagem de Águas Pluviais (SSDAP) é um sistema predial para captação de águas pluviais em grandes coberturas, acima de $5.000 \mathrm{~m}^{2}$ e pé-direito acima de $3,5 \mathrm{~m}$, que ainda é pouco estudado entre os pesquisadores no Brasil. Este sistema opera por pressão negativa, segundo o principio de Bernoulli, e dos condutos forçados, enquanto que o Sistema Convencional de Drenagem de Águas Pluviais (SCDAP) opera segundo os princípios dos condutos livres. Os componentes de um SSDAP são mostrados na Figura 1.

FIGURA 1 - Sistema sifônico - Principais componentes

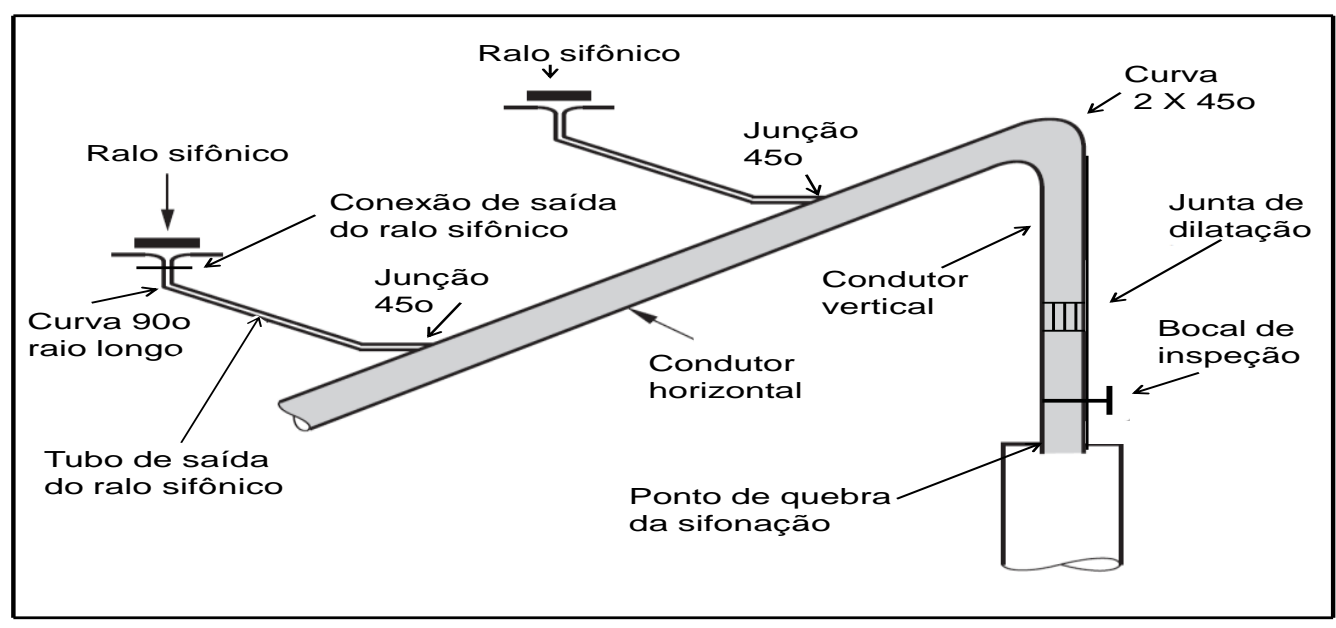

Fonte: Adaptado de BS 8490 (2007).

Estudos realizados por Richers (2018) com base nas publicações de May (1997), Bramhall e Saul (1998), Sommerhein (1999), Arthur e Swaffield (1999), Bowler e Arthur (1999), Arthur e Swaffield (2001), Rattenbury (2001), May (2004), Arthur e Wright (2007) permitem afirmar que o SSDAP poderá oferecer as seguintes vantagens em relação ao SCDAP: (i) quantidade menor de prumadas; (ii) menor quantidade de caixas de passagem; (iii) menor diâmetro dos tubos nas prumadas; (iv) drenagem de calhas internas sem utilizar caixas de passagem e (v) tubulação sem inclinação.

Este artigo apresenta o desenvolvimento histórico do surgimento das normas e diretrizes internacionais de SSDAP's. A comparação dos principais parâmetros normativos internacionais é detalhada na tabela 1. Somente a norma VDI 3806:2000 apresenta um procedimento de cálculo manual que não requer o uso de software, permitindo aos projetistas um cálculo independente dos coeficientes estabelecidos pelos fabricantes.

\section{REVISÃO BIBLIOGRÁFICA}

Os SSDAP's foram estudados a partir de 1970, mas somente começaram a ser largamente utilizados a partir de 1990 (Bramhall, 2005). Com a difusão da nova tecnologia, surgiu também a necessidade do estabelecimento de normas e diretrizes para o projeto, instalação e operação deste sistema sifônico. 
Nota-se, até hoje, que apenas alguns países possuem normas e diretrizes específicas para os SSDAP's, estes são: o Reino Unido, a Alemanha, os Estados Unidos da América e a Austrália.

As normas e diretrizes de drenagem de águas pluviais normalmente são subdivididas em três tipos de normas, como ocorre no Reino Unido e Alemanha: (i) norma e diretriz de instalações prediais de águas pluviais para edificações residenciais como casas e edifícios; (ii) norma e diretriz para drenagem externa, ou seja, para os terrenos e as áreas que não fazem parte das edificações; (iii) norma e diretriz para sistemas sifônicos de drenagem de águas pluviais.

Muitos países, como os Estados Unidos e a Austrália adotaram procedimentos diferentes. Nos Estados Unidos, diversos orgãos governamentais e associações de classe como a American Society of Mechanical Engineers (ASME), American Society of Plumbing Engineers (ASPE) e American Society For Testing Materials (ASTM) estabeleceram recomendações para projeto e instalação, agrupadas em diretrizes com validade em todo território norte-americano. A Austrália possui uma diretriz com recomendações técnicas válidas para todo território australiano e neozelandês (AU/NZ).

A ordem cronológica do surgimento das normas internacionais de SSDAP é visualizada na Figura 2.

FIGURA 2 - Cronologia das normas e diretrizes internacionais de SSDAP.

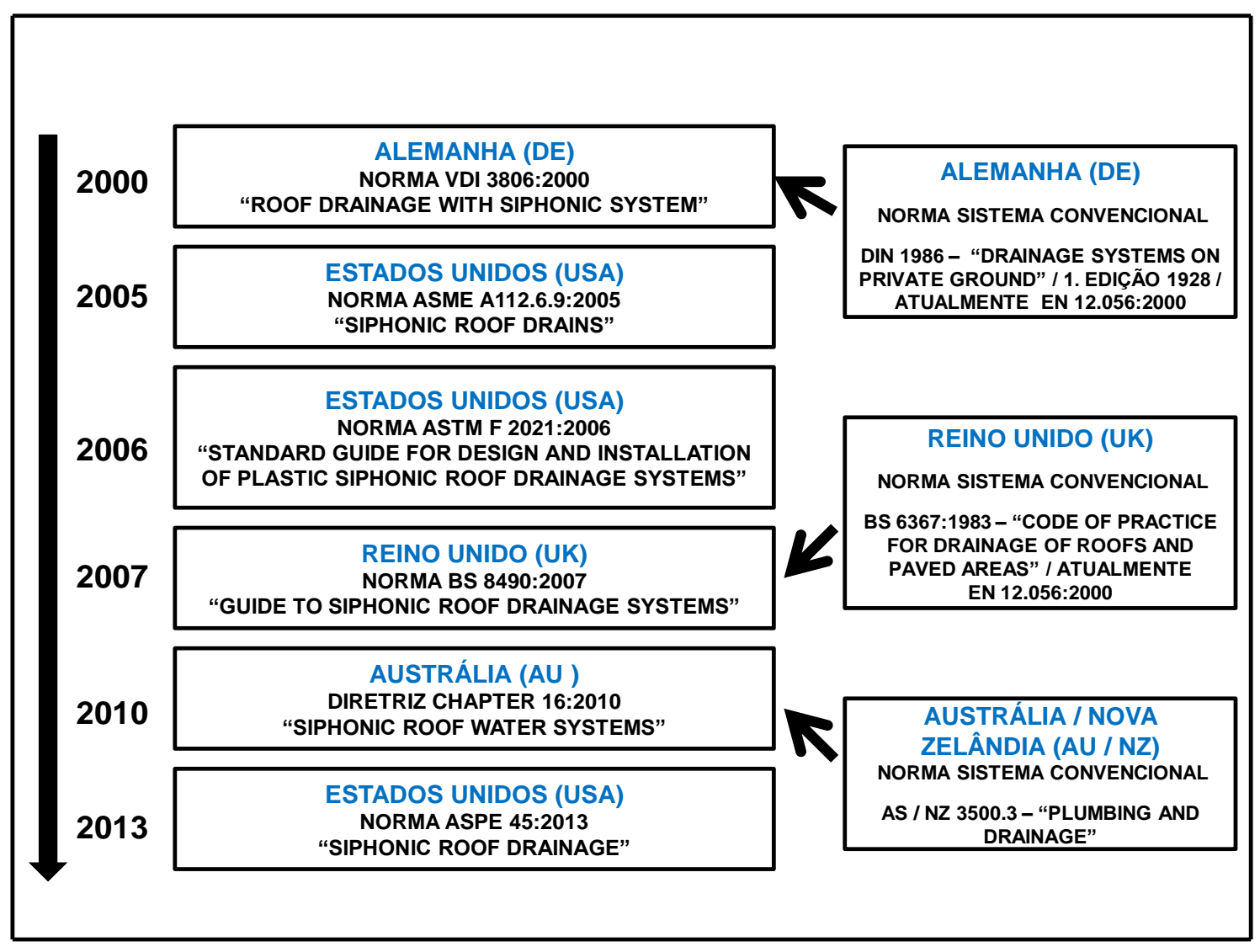

Fonte: Autores.

Observando-se a cronologia apresentada na Figura 2, verifica-se que nos países, Alemanha, Estados Unidos, Reino Unido e Austrália / Nova Zelândia, as normas para o 
SCDAP antecederam as normas para o SSDAP. No Brasil já existe uma norma para o SCDAP, a NBR 10844:1989, porém ainda não existe uma norma para a SSDAP.

\section{MÉTODO}

A pesquisa documental adotada para este trabalho permitiu identificar no site da DIN, ASPE, VDI e BSI, as normas existentes que apresentam os parâmetros para o método de dimensionamento do sistema sifônico de drenagem de águas pluviais, possibilitando seu estudo comparativo.

O estudo comparativo destas normas permitiu que fossem selecionados os principais parâmetros de dimensionamento de um SSDAP.

\section{RESULTADOS}

Na Tabela 1, encontra-se uma comparação detalhada dos tópicos, subtópicos e itens das principais normas internacionais de SSDAP.

TABELA 1 - Principais parâmetros de dimensionamento e operação.

\begin{tabular}{|c|c|c|}
\hline Parâmetros & Valores & Fonte \\
\hline \multicolumn{3}{|l|}{ Projeto } \\
\hline Verificação da perda de carga por prumada & Máximo $10 \%$ ou $1,0 \mathrm{~m}$ & BS 8490:2007 / ASPE 45:2013 \\
\hline Fator de segurança & $10 \%$ na vazão do projeto & ASPE 45:2013 \\
\hline Pressão negativa & Máxima - 900 mbar & VDI 3806:2000 \\
\hline Condutor vertical & $\begin{array}{l}\text { Comprimento mínimo } 4,0 \text { m; diâmetro } \\
\text { igual ou menor do que o tubo horizontal; } \\
\text { apresentar no topo duas curvas } 450\end{array}$ & $\begin{array}{l}\text { VDI 3806:2000 / ASPE 45:2013 / } \\
\text { BS 8490:2007 }\end{array}$ \\
\hline Tubo de saída do ralo sifônico & $\begin{array}{l}\text { Mínimo } 1.0 \text { m de comprimento, curva } \\
90 \text { o na parte inferior }\end{array}$ & VDI 3806:2000 / ASPE 45:2013 \\
\hline Velocidades no sistema sifônico & $\begin{array}{l}\text { Tubos, mínimo 1,0 m/s, máximo } 6-7 \\
\text { m/s, tubo vertical de saída, 2,5 - 3,0 m/s }\end{array}$ & $\begin{array}{l}\text { VDI 3806:2000 / ASPE 45:2013 / } \\
\text { BS 8490:2007 }\end{array}$ \\
\hline Tempo de enchimento & Máximo $60 \mathrm{~s}$ & BS 8490:2007 \\
\hline \multicolumn{3}{|l|}{ Calhas } \\
\hline Inclinação e tamanho & $\begin{array}{l}\text { Até } 1 \% \text { de inclinação, capacidade da } \\
\text { vazão de projeto }\end{array}$ & ASPE 45:2013 / BS 8490:2007 \\
\hline \multicolumn{3}{|l|}{ Ralos Sifônicos } \\
\hline Posicionamento & $\begin{array}{l}\text { Distância máxima } 20 \mathrm{~m} \text {, equidistante um } \\
\text { do outro e mínimo de dois ralos por } \\
\text { calha }\end{array}$ & VDI 3806:2000 / BS 8490:2007 \\
\hline \multicolumn{3}{|l|}{ Sistemas secundários ou extravasores } \\
\hline Segurança & $\begin{array}{l}\text { Exigência de extravasor ou sistema } \\
\text { secundário com vazão adequada }\end{array}$ & BS 8490:2007 / Chapter 16:2010 \\
\hline \multicolumn{3}{|l|}{$\underline{\text { Tubulação }}$} \\
\hline Espessura das paredes & Mínimo para PEAD é Schedule 40 & ASPE 45:2013 / ASTM F 2021:2006 \\
\hline \multicolumn{3}{|l|}{ Operação } \\
\hline Limpeza e caixas de passagem & $\begin{array}{l}\text { Mínimo } 3 \text { - } 4 \text { vezes / ano; caixas de } \\
\text { passagem com grelha para ventilação }\end{array}$ & BS 8490:2007 / ASPE 45:2013 \\
\hline
\end{tabular}

Fonte: Autores. 
Os parâmetros da Tabela 1 serão comentados resumidamente a seguir.

- Projeto - Informações gerais

No projeto de um SSDAP é importante adotar um fator de segurança de $10 \%$ na vazão de projeto, não ultrapassar a pressão negativa máxima de (-) 900 mbar, respeitar o tempo de enchimento de no máximo 60s, atentar para as velocidades mínimas da água nos tubos, de 1,0 m/s e no condutor vertical, na saída do SSDAP, de 2,5 - 3,0 m/s. O comprimento mínimo do tubo de saída do ralo sifônico deve ser de $1,0 \mathrm{~m}$ e condutor vertical de saída do SSDAP 4,0 m. A perda de carga por prumada em relação à carga disponível não deve ultrapassar $10 \%$ ou (-) 1,0 m.

- Calhas - Informações gerais

Recomenda-se uma inclinação de até $1 \%$ na calha, além da calha possuir a capacidade da vazão de projeto do SSDAP.

- Ralos sifônicos - Informações gerais

Os ralos sifônicos devem estar posicionados na calha equidistantes um do outro, não ultrapassando a distância de $20 \mathrm{~m}$.

- Sistemas secundários ou extravasores - Informações gerais

É exigida a instalação de extravasor ou sistema secundário, como medida de segurança.

- Tubulação - Informações gerais

Tubos em PEAD devem apresentar no mínimo paredes de espessura de Schedule 40.

- Operação - Informações gerais

As manutenções de limpeza devem ser de quatro vezes no primeiro ano de operação. A primeira caixa de passagem após o término de um SSDAP deve apresentar grelha com ventilação.

Os autores concluem, após análise dos parâmetros, descritos nas normas, que o projeto de um SSDAP deve ser visto como um conjunto de quatro grupos de informações denominadas fases A, B, C e D, que estão representados pela Figura 3.

FIGURA 3 - Sistema sifônico - Fases do projeto.

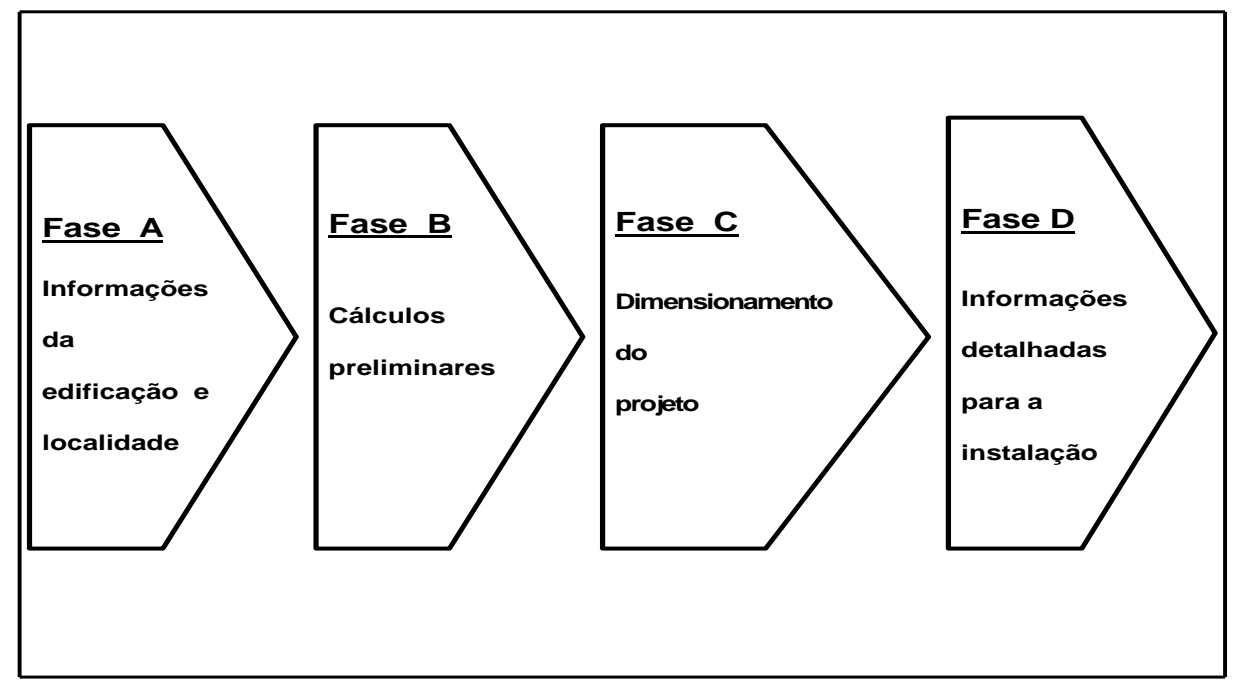

Fonte: Autores. 
A fase A é formada por informações específicas da edificação e localidade, tais como dimensões, calhas e precipitação pluviométrica. A fase B é composta de cálculos preliminares, como área da cobertura e vazão de projeto. A fase $\mathrm{C}$ envolve a aplicação das equações específicas decorrentes do Princípio de Bernoulli, envolvendo velocidades, diâmetros dos tubos, rugosidade e pressões negativas. Na fase $\mathrm{D}$ estão agrupadas as informações relevantes para a instalação de um SSDAP.

A maioria dos parâmetros descritos na Tabela 1 são utilizados na fase $C$, dimensionamento do projeto, da Figura 3.

\section{CONCLUSÃO}

As normas VDI 3806:2000, BS 8490:2007 e ASPE 45:2013 apresentam as principais equações para o cálculo e dimensionamento de um SSDAP.

A partir destas normas foi possível selecionar seis dos principais parâmetros. Estes, por sua vez, foram agrupados na fase $\mathrm{C}$ das quatro fases de um projeto de SSDAP.

Para auxiliar os projetistas de SSDAP no Brasil, a norma específica deveria contemplar os seis parâmetros principais destacados na Tabela 1: i) projeto; ii) calhas; iii) ralos sifônicos; iv) extravasores; v) tubulação e vi) operação.

\section{REFERÊNCIAS}

AMERICAN SOCIETY OF MECHANICAL ENGINEERS. ASME A112.6.9-2005. Siphonic roof drains. New York: ASME, 2005.

AMERICAN SOCIETY OF PLUMBING ENGINEERS. ASPE 45:2013: Siphonic roof drainage. Rosemont: ASPE, 2013.

AMERICAN SOCIETY FOR TESTING MATERIALS. ASTM F 2021-2006: Standard guide for design and installation of plastic siphonic roof drainage systems. West Conshohocken: ASTM, 2006.

ARTHUR, S.; SWAFFIELD, J. A. Understanding siphonic rainwater drainage systems. In: INTERNATIONAL SYMPOSIUM ON WATER SUPPLY AND DRAINAGE FOR BUILDINGS, 25., Edinburgh, 1999. Proceedings... Delf: CIB W062, 1999. B1.

ARTHUR,S.; SWAFFIELD, J. A. Siphonic roof drainage: current understanding. Urban Water, v. 3, p. 43-52, 2001.

ARTHUR, S.; WRIGHT, G. B. Siphonic roof drainage systems - priming focused design. Building and Environment, Edinburgh, v. 42, n. 6, p. 2421-2431, 2007.

ASSOCIAÇÃO BRASILEIRA DE NORMAS TÉCNICAS. NBR 10.844: Instalações prediais de águas pluviais. Rio de Janeiro: ABNT, 1989.

AUSTRALIAN GOVERNMENT. Departament of Planning and Local Government. Siphonic roofwater systems. In: Water sensitive urban design technical manual. Greater Adelaide Region: Government of South Austrália, 2010. Chapter 16.

BOWLER, R.; ARTHUR, S. Siphonic roof rainwater drainage - design considerations. In: INTERNATIONAL SYMPOSIUM ON WATER SUPPLY AND DRAINAGE FOR BUILDINGS, 25., 1999, Edinburgh. Proceedings... Delf: CIB W062, 1999. A 3. 
BRAMHALL, M. A. The performance of siphonic rainwater outlets within gutters. 2005. Thesis (PhD ) - University of Sheffield, 2005.

BRAMHALL, M. A.; SAUL, A. J. Examination of the performance of syphonic rainwater outlets. In: INTERNATIONAL SYMPOSIUM ON WATER SUPPLY AND DRAINAGE FOR BUILDINGS, 1998, Rotterdam. Proceedings... Delf: CIB W062, 1998.

BRITISH STANDARDS INSTITUTE. BS 8490:2007: Guide to siphonic roof drainage systems. London: BSI, 2007.

DEUTSCHES INSTITUT FÜR NORMUNG. EN 12.056-3: Schwerkraftentwässerungsanlagen innerhalb von Gebäuden. Teil 3: Dachentwässerung, Planung und Bemessung. Deutsche Fassung. EN 12.056-3:2000. DIN. Januar. 2001.

MAY, R. W. P. The design of conventional and siphonic roof-drainage systems. Journal CIWEM, 11, Feb. 1997.

MAY, R. W. P. Design criteria for siphonic roof drainage systems. Wallingford: HRS, 2004. (Report SR 654).

RICHERS,S.S. Sistema sifônico de drenagem de águas pluviais em grandes coberturas - Estudo de caso. 2018. Dissertação de Mestrado - IPT.

RATTENBURY, J. Fundamentals of siphonic roof drainage. PM Engineer, p. 52-58, Mar. 2001.

SOMMERHEIN, P. Design parameters for roof drainage systems. In: INTERNATIONAL SYMPOSIUM ON WATER SUPPLY AND DRAINAGE FOR BUILDINGS, 25., 1999, Edinburgh. Proceedings... Delf: CIB W062, 1999. A4.

VEREIN DEUTSCHER INGENIEURE. VDI 3806:2000: Roof drainage with siphonic system. April 2000. 